Article

\title{
Study on the Changes of Structures and Properties of PAN Fibers during the Cyclic Reaction in Supercritical Carbon Dioxide
}

\author{
Mengmeng Qiao ${ }^{1}{ }^{\mathbb{D}}$, Haijuan Kong ${ }^{2, *}$, Xiaoma Ding ${ }^{1}$, Zhifeng Hu ${ }^{1}$, Luwei Zhang ${ }^{1}$, \\ Yuanzhi Cao ${ }^{1}$ and Muhuo $\mathrm{Yu}^{1, *}$ \\ 1 State Key Laboratory for Modification of Chemical Fibers and Polymer Materials, College of Materials \\ Science and Engineering, Donghua University, Shanghai 201620, China; 2160320@mail.dhu.edu.cn (M.Q.); \\ 1159124@mail.dhu.edu.cn (X.D.); 2160226@mail.dhu.edu.cn (Z.H.); 2170293@mail.dhu.edu.cn (L.Z.); \\ 2180410@mail.dhu.edu.cn (Y.C.) \\ 2 School of Materials Engineer, Shanghai University of Engineer Science, Shanghai 201620, China \\ * Correspondence: Konghaijuan@sues.edu.cn (H.K.); yumuhuo@dhu.edu.cn (M.Y.); \\ Tel.: +86-0216-7792904 (H.K. \& M.Y.)
}

Received: 26 January 2019; Accepted: 19 February 2019; Published: 1 March 2019

\begin{abstract}
Thermal pre-oxidation of polyacrylonitrile (PAN) fibers is a time-consuming and energy-consuming step in the production of PAN-based carbon fibers. In this paper, the effect of temperature on the structures and properties of PAN fibers cyclized in the supercritical carbon dioxide $\left(\mathrm{Sc}-\mathrm{CO}_{2}\right)$ medium was studied. The thermal behaviors of the PAN fibers were investigated by Fourier transform infrared spectra (FT-IR), X-ray diffraction (XRD), differential scanning calorimeter (DSC) and thermogravimetric analysis (TGA). The cyclization reaction was sensitive to the heating temperature and gas atmosphere. The FT-IR results of the PAN fibers treated in the $\mathrm{Sc}_{\mathrm{c}} \mathrm{CO}_{2}$ confirmed that the degree of cyclization increased with the increase of the cyclization temperature. Compared with the PAN fibers treated in the air, the PAN fibers treated in the $\mathrm{Sc}-\mathrm{CO}_{2}$ showed a higher degree of cyclization even at the same temperature. These findings might be related to the osmotic action of Sc- $\mathrm{CO}_{2}$ causing the fibers to be further arranged in a regular manner, which was favorable for the cyclization reaction. Moreover, as one kind of high diffusion and high heat transfer media, the heat release during the cyclization of PAN fibers could be quickly removed by $\mathrm{Sc}-\mathrm{CO}_{2}$, which achieved the progress of the rapid-entry cyclization reaction.
\end{abstract}

Keywords: polyacrylonitrile fibers; supercritical carbon dioxide; thermal pre-oxidation; cyclization reaction

\section{Introduction}

Carbon fibers ( $\mathrm{CFs})$, as one of the new materials of lightweight high-performance fibers for composites, are defined as fibers containing at least $92 \mathrm{wt} \%$ carbon [1,2]. Owing to the advantages of excellent tensile properties, low densities, and high thermal and chemical stabilities, CFs are widely used in various fields [3-7]. CFs are produced from many different precursors, such as polyacrylonitrile (PAN), mesophase pitch, rayon, etc. [8-10], among which PAN-based CFs are the preferred reinforcements for structural composites owing to their excellent strength and stiffness combined with the fact they are lightweight and cost less. The majority of CFs (about 95\%) are made from PAN fibers [11,12]. However, PAN-based CFs are not easy to commercialize due to the time-consuming pre-oxidation step, which will significantly increase the manufacturing cost. Therefore, the advanced processing technologies that aim to reduce the production cost of CFs should be developed. 
Different methods have been studied to accelerate the pre-oxidation rate of PAN fibers, such as ultraviolet (UV) and gamma rays. Marlon et al. [13] added the addition of one kind of photo-initiator during the spinning process and then induced cyclization and crosslinks in PAN fibers with UV at low temperatures. However, excessive radiation could cause defects in the fibers and it was not easy to control the dose of radiation. Zhao et al. [14] studied the treatment of PAN fibers with different pre-oxidation degrees by gamma rays, followed by carbonization. However, it was necessary to accurately control the amount of gamma radiation. Zhao et al. [15] carried out pre-oxidation experiments over different temperature ranges. This method could obtain CFs with higher mechanical properties but the pre-oxidation time was too long. Lin Jianhua et al. [16] conducted the pre-oxidation experiment of PAN fibers with microwave assistance. However, the cost of the microwave equipment was relatively high, and the speed of pre-oxidation was not well controlled.

The thermal pre-oxidation process was one of the most time-consuming and energy-consuming steps $[17,18]$. In recent years, great interest has been given to the dyeing of synthetic fibers in $\mathrm{Sc}-\mathrm{CO}_{2}$ with the aim to achieve their eco-friendly production by manipulating the dyeing temperature, pressure, and time [19-21]. During the supercritical dyeing process, $\mathrm{Sc}-\mathrm{CO}_{2}$ fluid near or slightly above its critical points $\left(\mathrm{T}_{\mathrm{C}}=31.10^{\circ} \mathrm{C}, \mathrm{P}_{\mathrm{C}}=7.38 \mathrm{MPa}\right)$ produced substantial polymer swelling because of the dissolution of $\mathrm{CO}_{2}$ in polymers [18]. Therefore, $\mathrm{Sc}-\mathrm{CO}_{2}$ was used as a non-toxic solvent in extractions [22,23], separations [24], chemical reactions [25], and various other applications [26,27]. Furthermore, $\mathrm{CO}_{2}$ gives superior heat transfer properties for near-critical operation. From Table 1, we can obtain the thermal conductivity $(\lambda)$ of Sc- $-\mathrm{CO}_{2}$ and air under different conditions, from which we can observe that the $\lambda$ of $\mathrm{Sc}-\mathrm{CO}_{2}$ is significantly higher than that of the air, indicating that $\mathrm{Sc}-\mathrm{CO}_{2}$ can be used as a good heat transfer medium to transfer the heat released during the cyclization reaction.

Table 1. Summary of the thermal conductivity data of supercritical carbon dioxide $\left(\mathrm{Sc}-\mathrm{CO}_{2}\right)$ and air in different studies.

\begin{tabular}{|c|c|c|c|c|}
\hline Medium & Reference & $T(K)$ & $\mathrm{P}(\mathrm{MPa})$ & $\lambda\left(\mathrm{mW} \cdot \mathrm{m}^{-1} \cdot \mathrm{K}^{-1}\right)$ \\
\hline \multirow{4}{*}{$\mathrm{Sc}-\mathrm{CO}_{2}$} & Johns et al. [28] & $381.07-473.38$ & $8.32-30.6$ & $30.98-61.57$ \\
\hline & Le Neindre et al. [29] & $311.25-960.85$ & $7.6-127.8$ & $27.7-166.0$ \\
\hline & Michels et al. [30] & $313.2-348.29$ & $8.437-209.68$ & 30-194.81 \\
\hline & Le Neindre [31] & $326.65-951.15$ & $10-120$ & $30.8-154.4$ \\
\hline Air & Stephan et al. [32] & $373.0-573.0$ & / & $9.359-26.35$ \\
\hline
\end{tabular}

This study aims to find a new way to use $\mathrm{Sc}_{-}-\mathrm{CO}_{2}$ as a kind of medium to quickly remove the heat released during the cyclization to accelerate the progress of the cyclization and reduce the production cost of CFs. We can see the structural changes of PAN fibers during the cyclization and dehydrogenation from Figure 1.

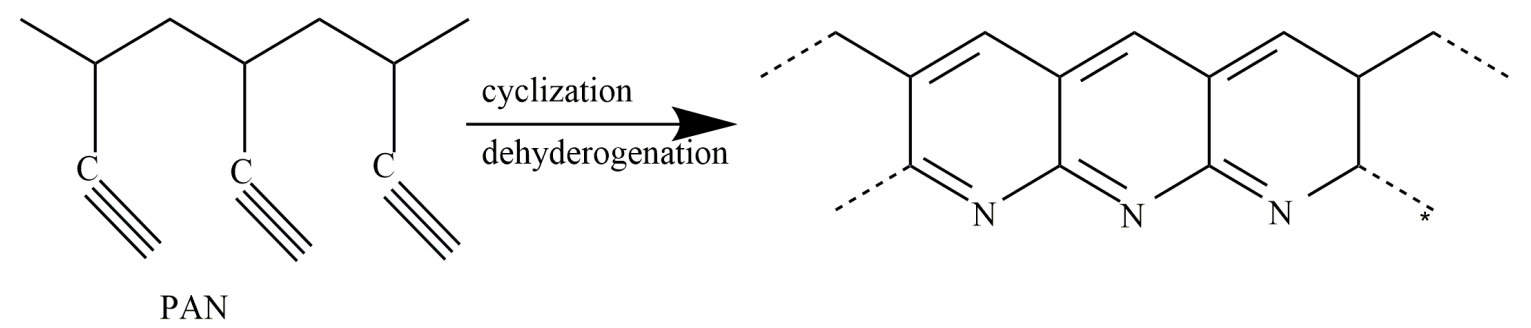

Figure 1. Schematic diagram of cyclization and dehydrogenation of polyacrylonitrile (PAN) fibers. 


\section{Materials and Methods}

\subsection{Materials and Sample Preparation}

PAN fibers (24,000 filaments per fiber bundle) were wet-spun from a copolymer of acrylonitrile/ acrylamide/methyl acrylate and supplied by Weihai Fiber Development Co., Ltd., Weihai, China. Carbon dioxide with a purity of $99.99 \%$ was supplied by Sinopharm Chemical Reagent Co., Ltd., Shanghai, China.

The reactor was heated to a certain temperature, and then the PAN fibers were fixed on a stirrer in the reaction vessel. The $\mathrm{Sc}-\mathrm{CO}_{2}$ reactor was closed, the air in the reactor was removed with $\mathrm{CO}_{2}$, and then the $\mathrm{CO}_{2}$ was passed into the reactor and reached a certain pressure. After a period of reaction, the $\mathrm{CO}_{2}$ was removed, the fibers were taken out, and the pre-oxidized fibers we needed were obtained.

\subsection{Characterizations}

Fourier transform infrared spectra (FT-IR) was recorded on the Nicolet 6700 FT-IR spectrophotometer ((Thermo Fisher Company, New York, NY, USA) using the method of KBr troches with a scanning wavenumber range of $400-4000 \mathrm{~cm}^{-1}$. The relative cyclization rate (RCR) of PAN fibers was calculated according to Equation (1):

$$
R C R=\frac{I_{\mathrm{C}=\mathrm{N}}}{I_{\mathrm{C}=\mathrm{N}}+I_{\mathrm{C} \equiv \mathrm{N}}} \times 100 \%
$$

where $I_{\mathrm{C}=\mathrm{N}}$ : transmission peak intensity of $\mathrm{C}=\mathrm{N} ; I_{\mathrm{C} \equiv \mathrm{N}}$ : transmission peak intensity of $\mathrm{C} \equiv \mathrm{N}$.

$\mathrm{X}$-ray diffraction (XRD) studies of the as-received fibers and pre-oxidized PAN fiber bundles were carried out on the Bruker D/max 2550 VB (Bruker Co., Kanagawa, Japan). The different pre-oxidation fibers and the as-received were all cut into powders before testing using XRD. The range of the scan angle was from $5^{\circ}$ to $60^{\circ}$ with a step width of 0.02 . The aromatic index (AI) of PAN fibers was calculated according to Equation (2):

$$
A I=\frac{I_{\mathrm{c}}}{I_{\mathrm{c}}+I_{\mathrm{p}}} \times 100 \%
$$

where $I_{\mathrm{c}}$ : diffraction intensity of PAN fibers at $2 \theta=29^{\circ}, I_{\mathrm{p}}$ : diffraction intensity of PAN fibers at $2 \theta=16.5^{\circ}$.

Thermal behaviors of pre-oxidation fibers and the as-received fibers were studied using a differential scanning calorimeter (DSC Q100, TA Instrument, Shanghai, China) using a heating rate of $10{ }^{\circ} \mathrm{C} \cdot \mathrm{min}^{-1}$ in a nitrogen atmosphere; the temperature was varied from 30 to $400{ }^{\circ} \mathrm{C}$. The cyclization degree (CD) of PAN fibers was calculated according to Equation (3):

$$
C D=\frac{H_{\mathrm{U}}-H_{0}}{H_{\mathrm{U}}} \times 100 \%
$$

where $H_{\mathrm{U}}$ : heat released from as received fibers, $H_{0}$ : heat released from PAN fibers treated in different temperatures.

The thermal stability of the fibers was investigated by the Thermogravimetric analysis (TGA) (TG 209 F1 Netzsh, Shanghai, China). The ramp rate was $10^{\circ} \mathrm{C} \cdot \mathrm{min}^{-1}$, and the curves were recorded under a working $\mathrm{N}_{2}$ flux equal to $50 \mathrm{~mL} \cdot \mathrm{min}^{-1}$. The temperature range was employed from room temperature to $900{ }^{\circ} \mathrm{C}$ during the test.

The changes in the density of the PAN fibers treated in different conditions were measured by a liquid weight balance (PZ-B-5, Shanghai, China) using the floatation method. After drying, the PAN fibers and the as-received fibers were cut into $0.5 \mathrm{~mm}$ or less, and then these were placed in the formulated equilibrium solution which was a mixture of carbon tetrachloride and N-heptane. The proportions of the heavy liquid and the light liquid were adjusted to suspend the fibers until the fibers were evenly distributed in the mixture and remained suspended for more than $4 \mathrm{~h}$. 
The densitometer was used to determine the density of solution, which was also the density of the PAN fibers.

\section{Results and Discussion}

\subsection{FT-IR Evolution Analysis of the PAN Fibers Heated at Different Temperatures}

It was suggested that both the shrinkage and decrease in the nitrile group concentration during the pre-oxidation process could be used to monitor the process of pre-oxidation. Figure 2 showed the changes of the infrared spectra of PAN fibers heated at different temperatures in the air and $\mathrm{Sc}-\mathrm{CO}_{2}$. The peak at $2240 \mathrm{~cm}^{-1}$ in the infrared spectra was assigned to the $-\mathrm{C} \equiv \mathrm{N}$ stretching of the acrylonitrile unit in the polymer chains. The other absorption peaks in the polymer were given as follows: The double bond stretching vibration zone $\left(1690-1500 \mathrm{~cm}^{-1}\right), \mathrm{C}-\mathrm{H}$ stretching vibration zone $\left(1475-1000 \mathrm{~cm}^{-1}\right)$. Figure $2 \mathrm{a}$ showed the spectra of as-received and treated PAN fibers in Sc- $\mathrm{CO}_{2}$. Comparing the untreated PAN fibers with pre-oxidized PAN fibers treated in the $\mathrm{Sc}-\mathrm{CO}_{2}$, the absorption at $2240 \mathrm{~cm}^{-1}$ showed a reduction for PAN fibers treated at $140{ }^{\circ} \mathrm{C}$ in Sc- $\mathrm{CO}_{2}$, matching with an increase in the intensity of the absorption band at $1600 \mathrm{~cm}^{-1}$. The results showed that the $-\mathrm{C} \equiv \mathrm{N}$ bond of the PAN fibers decreased after the heat treatment, and the intensity of the vibrational peak of $\mathrm{C}=\mathrm{N}$ increased, which also indicated that the ring structures in PAN fibers have been produced after treatment at $140{ }^{\circ} \mathrm{C}$. Further information was also given in the FT-IR spectra: The intensity of the vibrational peak at $1453 \mathrm{~cm}^{-1}\left(\mathrm{C}-\mathrm{H}_{2}\right)$ gradually weakened, and the intensity of the vibrational peak at $1377 \mathrm{~cm}^{-1}(\mathrm{C}-\mathrm{H})$ gradually increased. All the changes in the FT-IR spectra indicated that more complex structures have been formed during the cyclization reaction process. Comparing the infrared spectra of PAN fibers treated at different temperatures, with the increase of treatment temperature, the stretching vibration peaks at 1600 and $1377 \mathrm{~cm}^{-1}$ gradually increased, while the stretching vibration peak at 2240 and $1453 \mathrm{~cm}^{-1}$ gradually weakened. These results indicated that the crosslinked and cyclized structures in PAN fibers increased gradually with the increase of temperature, and accordingly, the degree of cyclization increased with the increase of the cyclization temperature.
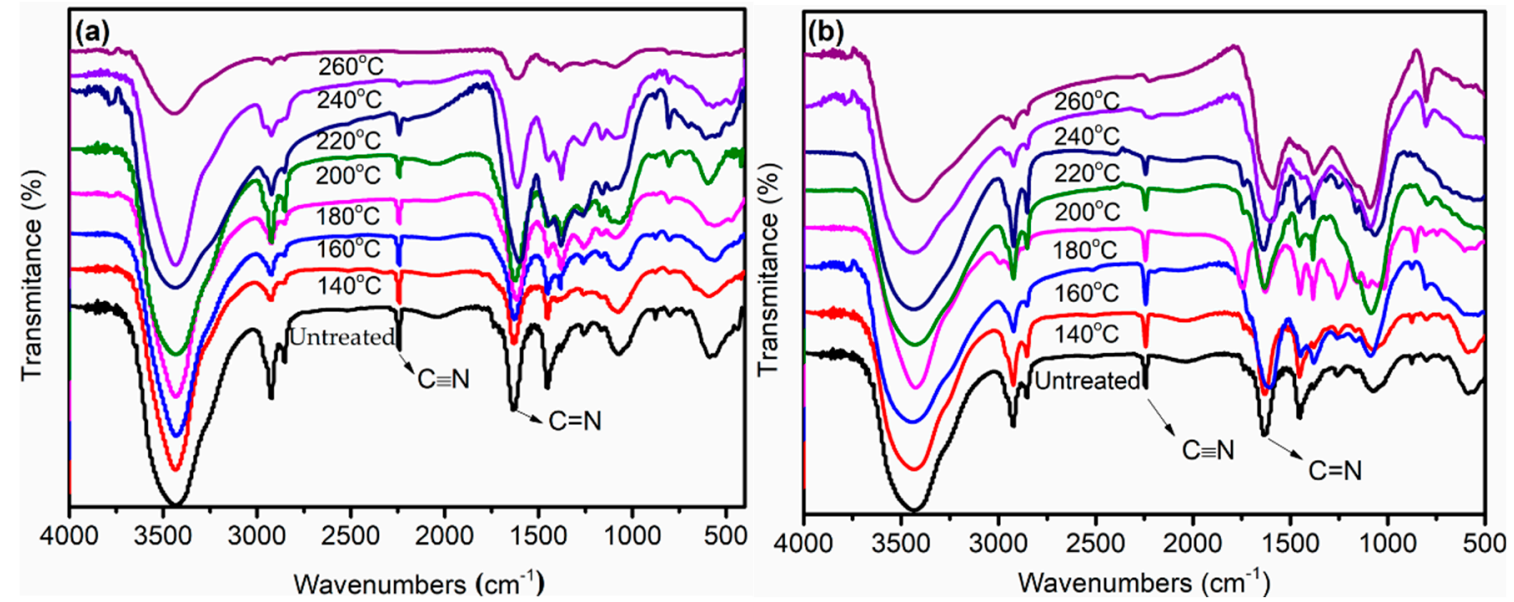

Figure 2. Fourier transform infrared spectra (FT-IR) spectra of PAN fibers treated at different temperatures: (a) in $\mathrm{Sc}-\mathrm{CO}_{2} ;(\mathbf{b})$ in air.

The effect of temperature on the cyclization reaction in the air was consistent with the trend in Sc- $\mathrm{CO}_{2}$. Comparing the two FT-IR spectra in Figure 2, the changes of absorption spectra at 2240 and $1600 \mathrm{~cm}^{-1}$ were more obvious in $\mathrm{Sc}-\mathrm{CO}_{2}$, indicating that the pre-oxidized PAN fibers treated in $\mathrm{Sc}-\mathrm{CO}_{2}$ showed a higher degree of cyclization than the PAN fibers obtained in the air, even at the same temperature. The RCR could be calculated using Equation (1). The RCR of fibers treated at different temperatures shown in Figure 3 also indicated that the PAN fibers had a higher degree of cyclization 
treated in $\mathrm{Sc}-\mathrm{CO}_{2}$ than the PAN fibers treated in air. It might be due to the fact that $\mathrm{Sc}-\mathrm{CO}_{2}$, as a kind of fluid, was more likely to carry away the heat released by the cyclization of PAN fibers, thereby accelerating the cyclization of PAN fibers.

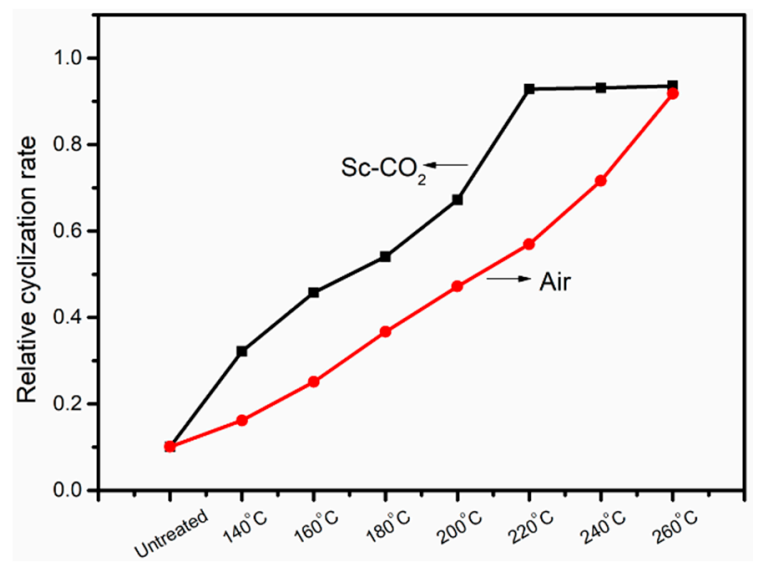

Figure 3. The relative cyclization rate (RCR) of PAN fibers calculated by FT-IR spectra.

\subsection{X-ray Diffraction Analysis of PAN Fibers Heated at Different Temperatures}

Figure 4 depicts the XRD patterns of PAN fibers treated at different temperatures in air and $\mathrm{Sc}-\mathrm{CO}_{2}$. The XRD patterns showed the typical diffraction peaks at $2 \theta=16.5^{\circ}$ and $2 \theta=29.0^{\circ}$ of PAN fibers, reflecting the ordered crystal structure made up of PAN linear macromolecules. There was a strong diffraction peak at $2 \theta=16.5^{\circ}$ corresponding to the crystal plane (100), which reflected the spacing of the molecular chains. In addition, there was another weak diffraction peak near $2 \theta=29.0^{\circ}$ corresponding to the crystal plane (110), which reflected the distance between nearly parallel molecular pieces. A wide diffuse reflection region existed between the two diffraction peaks at $2 \theta=16.5^{\circ}$ and $29.0^{\circ}$, indicating that the disordered phase was distributed throughout the structure in a non-discrete manner. Due to the cyclization, the structure of as-received fibers was very different from the pre-oxidized fibers; therefore, we could judge the degree of cyclization by the differences in the XRD spectra. As can be seen from the XRD patterns in Figure 4, the diffraction intensity $I_{p}$ of the peak at $2 \theta=16.5^{\circ}$ evidently decreased with the increase of heating temperature, matching with the decrease of the characteristic diffraction peak intensity $I_{\mathrm{c}}$ at $2 \theta=29.0^{\circ}$. When the temperature was $220^{\circ} \mathrm{C}$, the diffraction peak had almost disappeared, which indicated the crystal structure was basically no longer present. This experimental result was consistent with the reduction and conjugation of the $\mathrm{C} \equiv \mathrm{N}$ group, which had a predominant role in the change of the PAN molecule during the thermal pre-oxidation. This indicated that the molecular chains in PAN fibers tended to be disordered, which tended to reduce the difference in the ordered regularity between disordered and ordered intervals in the PAN two-phase structure proposed by Lin et al. [33]. These phenomena meant that $\mathrm{C} \equiv \mathrm{N}$ in the PAN molecule had reacted to produce $\mathrm{C}=\mathrm{N}$, which implied that cyclization and dehydrogenation started to occur in the PAN fibers. At the same time, the higher the treatment temperature, the higher the cyclization reaction degree.

The cyclization of as-received fibers was a reaction that destroyed orientation and decreased crystallinity, which was closely related to the crystallinity. The crystallinity of PAN fibers is given in Figure 5, from which we can observe that the crystallinity of PAN fibers treated in $\mathrm{Sc}-\mathrm{CO}_{2}$ was greatly reduced, that is to say, a large number of cross-network structures were produced. According to Equation (2), we could calculate the aromatic index (AI) of different pre-oxidation fibers, as shown in Figure 6. The AI of PAN fibers increased with the increase of experimental temperature, at the same time, the fibers treated in $\mathrm{Sc}-\mathrm{CO}_{2}$ had more cyclized structures than the fibers treated in air, even at the same temperature. These results showed that $\mathrm{Sc}-\mathrm{CO}_{2}$ fluid was useful to transfer the heat produced during the cyclization reaction and reduce the cyclization temperature to get PAN fibers with high cyclization. 

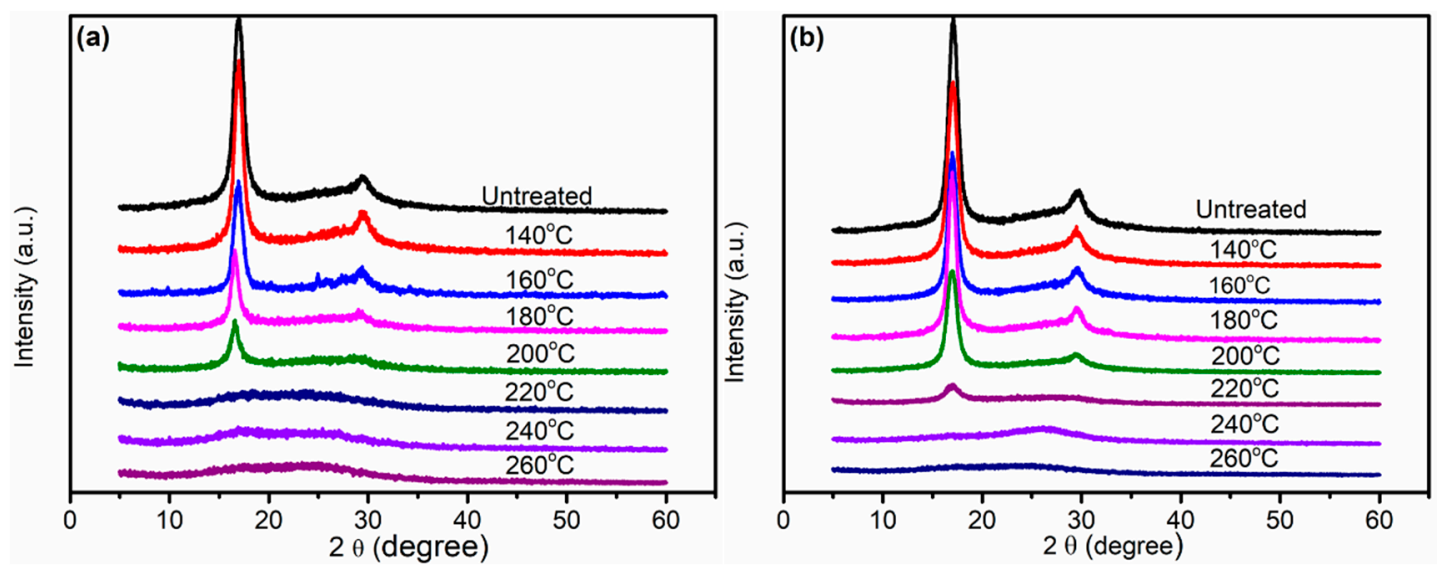

Figure 4. X-ray diffraction (XRD) patterns of PAN fibers treated at different temperatures: (a) in $\mathrm{Sc}^{-} \mathrm{CO}_{2}$; (b) in air.

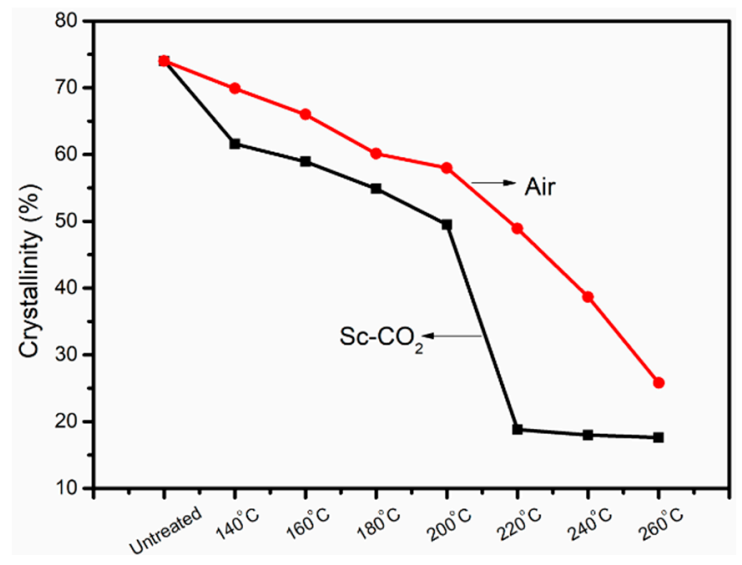

Figure 5. The crystallinities of PAN fibers treated in the air and $\mathrm{Sc}-\mathrm{CO}_{2}$.

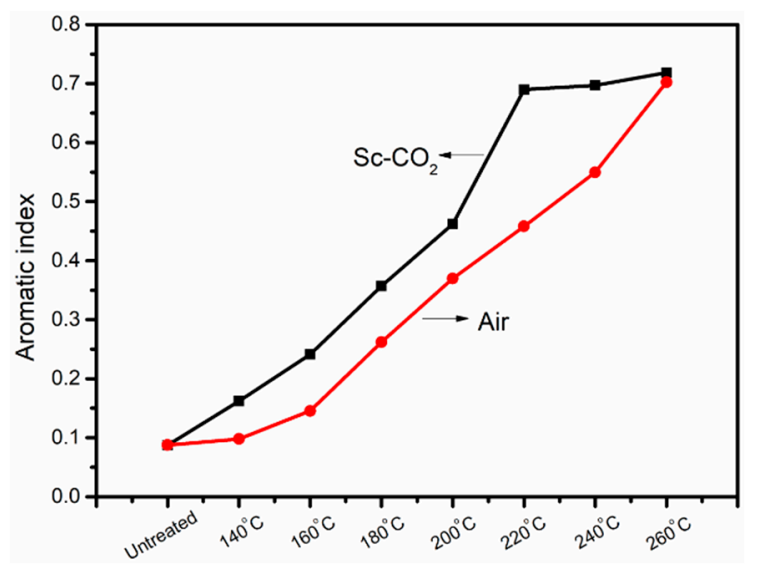

Figure 6. Aromatic Index of PAN fibers treated at different temperatures in air and $\mathrm{Sc}-\mathrm{CO}_{2}$.

\subsection{Differential Scanning Calorimeter Analysis of the PAN Fibers Heated at Different Temperatures}

Figure 7 displays the DSC curves of PAN fibers treated at different temperatures in air and Sc-CO The effect of heat temperatures on the degree of cyclization was mainly studied by DSC owing to the exothermic process during the cyclization reaction of PAN fibers. Due to the heat resistance of the ring structure, the cyclic structure of the PAN fibers hardly resulted in an exothermic reaction during the heating process of the PAN fibers, while the uncyclized structure underwent an exothermic reaction easily. Therefore, the degree of cyclization of PAN fibers could be calculated from the area of 
the exothermic peak during the heating process. As can be seen from Figures $7 \mathrm{a}$ and 8 , there was no obvious change in the area of the exothermic peak treated at $140^{\circ} \mathrm{C}$, but the area of the exothermic peak decreased gradually with the increase of the heat treatment temperature. The total exotherm amount of fibers gradually decreased, indicating that with the increase of the pre-oxidation temperature, the degree of cyclization increased and the heat release of the reaction decreased. At $220^{\circ} \mathrm{C}$, the peak area of the treated fibers clearly decreased, that is to say, the heat release decreased significantly, which indicated that the fibers had a high degree of cyclization. It could also be concluded that, as the temperature increased, the content of exotherm during heating gradually decreased and quantity of the aromatic cyclization structure increased.
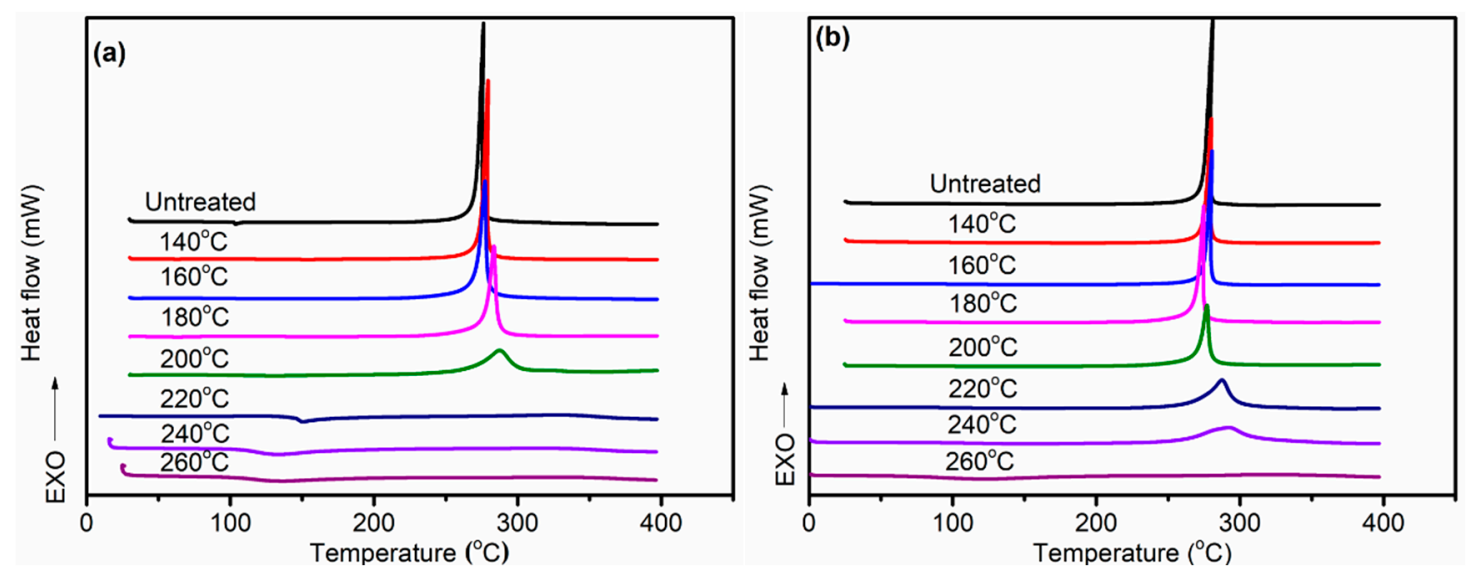

Figure 7. Differential scanning calorimetry (DSC) curves of PAN fibers treated at different temperatures: (a) in $\mathrm{Sc}-\mathrm{CO}_{2} ;(\mathbf{b})$ in air.

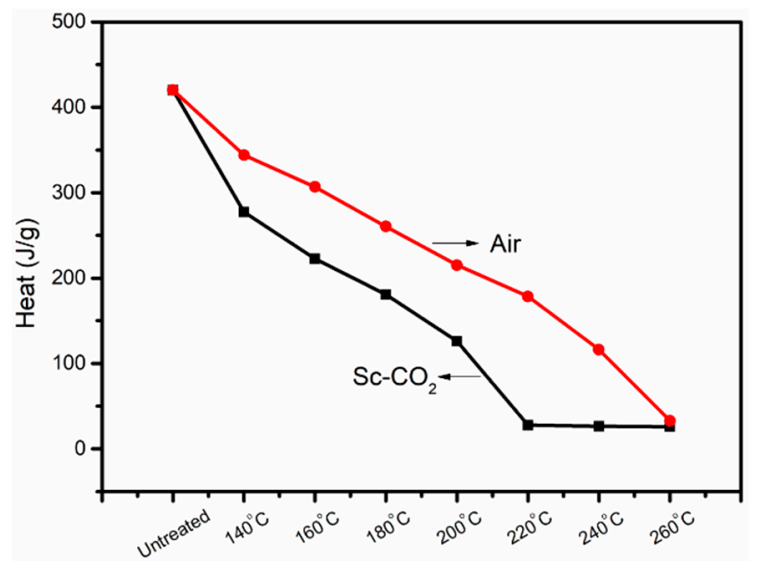

Figure 8. Exotherm of PAN fibers treated at different temperatures in air and $\mathrm{Sc}-\mathrm{SO}_{2}$.

Compared with DSC curves of fibers treated in air in Figure 7b, the exothermic peak of the fibers treated in $\mathrm{Sc}-\mathrm{CO}_{2}$ was significantly smaller even at the same temperature; therefore, it can be concluded that the fibers treated in $\mathrm{Sc}-\mathrm{CO}_{2}$ released less heat and had more aromatic cyclization structures. The CD of different PAN fibers was calculated according to Equation (3), as shown in Figure 9. We could interpret the results of experiments in the following sections: The cyclization reaction of PAN fibers was an exothermic process, so the heat released during the cyclization reaction should be taken away in time to prevent the fibers from being fused. The fibers treated in $\mathrm{Sc}-\mathrm{CO}_{2}$ had smaller exothermic peaks and more cyclized structures compared with the fibers treated in air at the same temperature. This was because $\mathrm{Sc}-\mathrm{CO}_{2}$ could enter into the inside of the fibers and the heat released by the PAN fibers during the cyclization was taken out in time to speed up the rate of reaction. 


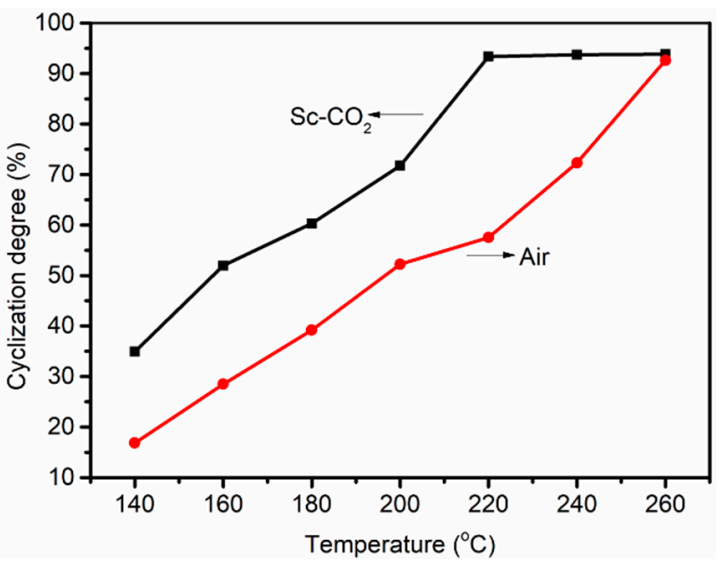

Figure 9. Cyclization degree of PAN fibers treated at different temperatures in air and $\mathrm{Sc}-\mathrm{SO}_{2}$.

\subsection{Thermogravimetric Analysis of PAN Polymers Heated at Different Temperatures}

The thermogravimetric (TG) curves and differential thermal gravity (DTG) curves of PAN fibers treated at different temperatures are shown in Figures 10 and 11, respectively. Due to the heat resistance of the ring structure, the cyclic structure of the PAN fibers hardly decomposed during the heating process of the PAN fibers, while the uncyclized structure was more prone to pyrolysis. When the heating temperature was constant, more heat-resistant aromatic cyclization structures were present in PAN fibers, the more difficult the thermal decomposition of fibers became, and the more thermal decomposition residue was obtained. Therefore, it was possible to obtain the degree of cyclization of the PAN fibers by the residual amount of the fibers after the decomposition. The mass of the fibers decreased at the beginning, resulting from the loss of moisture in the fibers. Comparing the pyrolytic residuals and decomposition curves of PAN fibers treated at different temperatures in Table 2 and Figure 10, it could be concluded that as the cyclization temperature increased, the quality of the fibers obtained was increased, which indicated that the amount of the ring structure resistant to decomposition in the cyclized fiber was increased with the increasing temperature. DTG curves showed that there were two decomposition peaks during the heat treatment process. Among them, the weight loss rate was the fastest in the $270-300{ }^{\circ} \mathrm{C}$ temperature range. From the DTG curve, we concluded that with the increase of the pre-oxidation temperature, the weight loss rate of the fibers in both temperature ranges is evidently slower, indicating that the content of the ring structures in the fiber was gradually increasing.
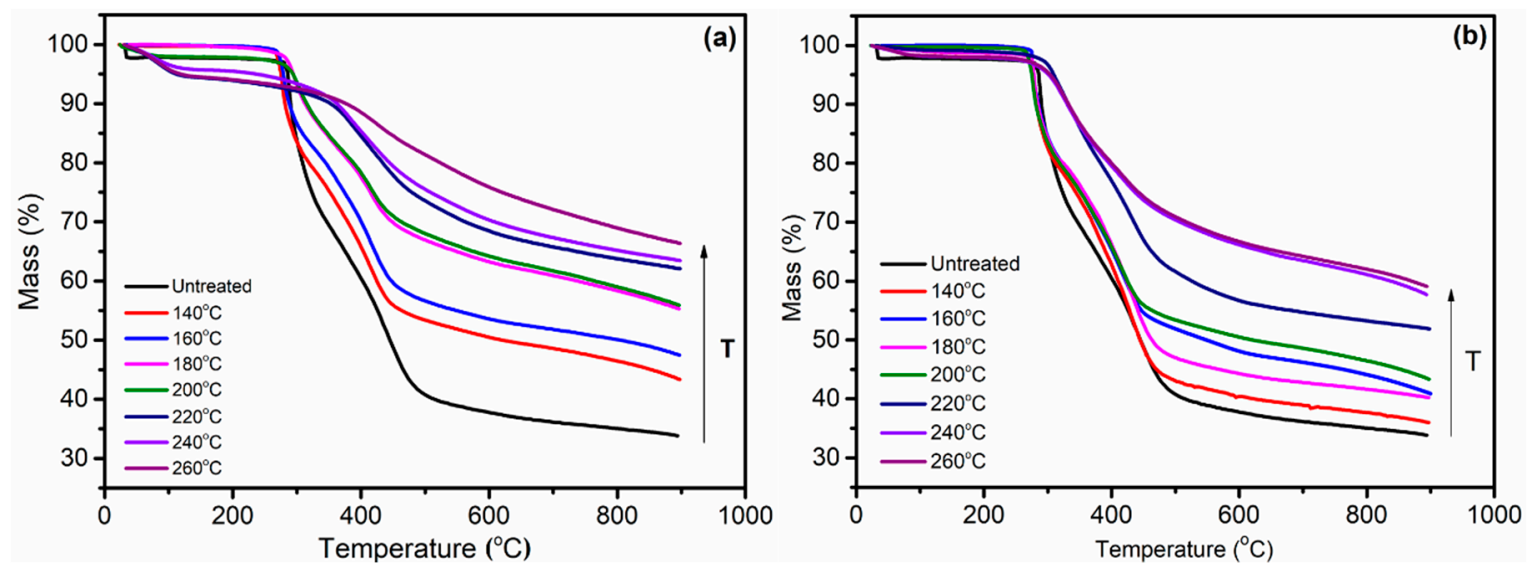

Figure 10. The thermogravimetric (TG) curves of PAN fibers treated at different temperatures: (a) in $\mathrm{Sc}-\mathrm{CO}_{2} ;(\mathbf{b})$ in air. 

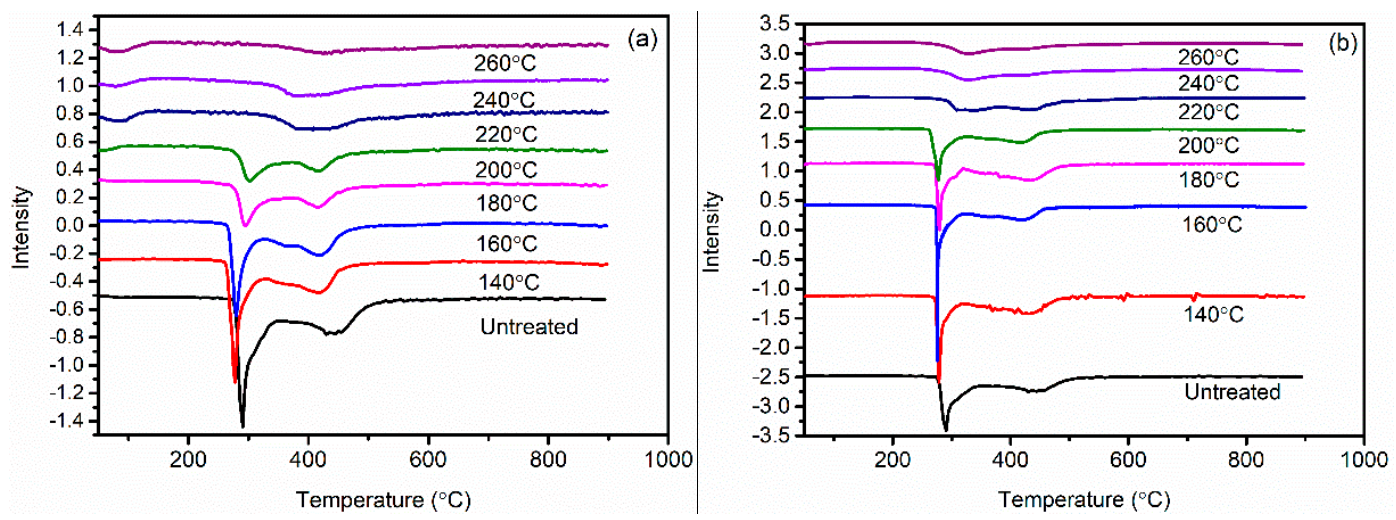

Figure 11. The differential thermal gravity (DTG) curves of PAN fibers treated at different temperatures: (a) in $\mathrm{Sc}-\mathrm{CO}_{2} ;(\mathbf{b})$ in air.

Table 2. Residual mass of the PAN fibers treated in $\mathrm{Sc}-\mathrm{CO}_{2}$ and air.

\begin{tabular}{ccc}
\hline Different Treatment Conditions & Residual Mass (\%)/air & Residual Mass (\%)/Sc-CO $\mathbf{C}_{\mathbf{2}}$ \\
\hline Untreated fibers & 33.54 & 33.54 \\
$140^{\circ} \mathrm{C}$ & 34.78 & 43.32 \\
$160^{\circ} \mathrm{C}$ & 43.11 & 47.43 \\
$180^{\circ} \mathrm{C}$ & 44.43 & 55.21 \\
$200^{\circ} \mathrm{C}$ & 46.21 & 55.80 \\
$220^{\circ} \mathrm{C}$ & 51.86 & 62.07 \\
$240^{\circ} \mathrm{C}$ & 61.36 & 63.45 \\
$260^{\circ} \mathrm{C}$ & 61.59 & 66.34 \\
\hline
\end{tabular}

At the same time, comparing the residual mass of the fibers obtained in the air with $\mathrm{Sc}-\mathrm{CO}_{2}$ at the same temperature, it could be concluded that the PAN fibers treated in the medium of Sc- $\mathrm{CO}_{2}$ had a greater residual quality than that in air, indicating a higher cyclization structure in PAN fibers treated in $\mathrm{Sc}_{-}-\mathrm{CO}_{2}$. This further confirmed the correctness of the experimental results of the previous FT-IR, XRD, and DSC. When the treatment temperature was $260^{\circ} \mathrm{C}$, the residual mass of PAN fibers treated in $\mathrm{Sc}-\mathrm{CO}_{2}$ was $66.34 \%$, which was much larger than the residual mass of $61.59 \%$ of PAN fibers treated in air, which indicated that the cyclization reaction in $\mathrm{Sc}-\mathrm{CO}_{2}$ might be a method to improve the carbon yield of CFs.

\subsection{Density Changes of PAN Fibers Treated in Different Conditions}

The density changes of pre-oxidized PAN fibers obtained at different temperatures are shown in Figure 12. The degree of cyclization and densities of PAN fibers had a close relationship owing to the following two reasons. The first reason was due to the effect of drawing to make its physical structure dense, the second was due to the occurrence of the cyclization and cross-linking reaction to increase the density. That is to say, the higher the density of PAN fibers, the higher the degree of cyclization in PAN fibers. It could be seen that as the temperature increased, the density of PAN fibers increased, which was consistent with the increase of the degree of cyclization calculated by the results of FT-IR, DSC, TGA, and XRD, indicating that the degree of cyclization constantly increased with the increasing temperature. Comparing the density of pre-oxidized PAN fibers treated in air with that of those treated in $\mathrm{Sc}-\mathrm{CO}_{2}$, we could obtain that the degree of cyclization of PAN fibers treated in $\mathrm{Sc}-\mathrm{CO}_{2}$ was higher than that in air at the same temperature. 


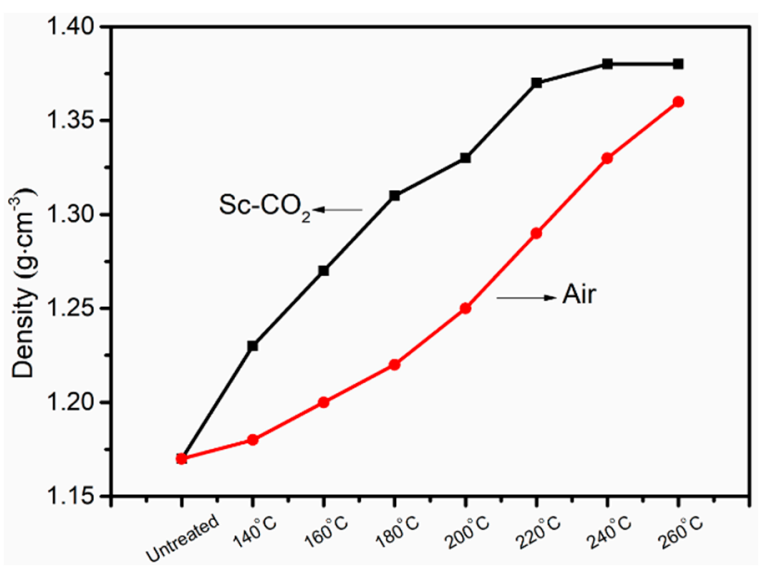

Figure 12. The density changes of PAN fibers.

It might be related to the osmotic action of $\mathrm{Sc}-\mathrm{CO}_{2}$. On the one hand, the action of $\mathrm{Sc}-\mathrm{CO}_{2}$ caused the fibers to be further arranged in a regular manner, which was favorable for the cyclization reaction. On the other hand, $\mathrm{Sc}-\mathrm{CO}_{2}$ is non-polar, and the physical properties can be easily tuned between the gas and liquid which can quickly remove the heat released during the cyclization of PAN, improving the progress of the cyclization reaction. Furthermore, the aromatic cyclization first occurred in the amorphous region, the PAN molecular segment in the amorphous region was more likely to move in $\mathrm{Sc}-\mathrm{CO}_{2}$, which was advantageous for the aromatic cyclization reaction. The aromatic cyclization reaction primarily caused an effective collision between the functional groups. The effective collisions formed among the functional groups in the macromolecular chains with a certain spatial position, a certain direction, and a certain angle. It required the molecular segments to have sufficient motion and soul to move, which could improve the opportunities for more effective collisions.

\section{Conclusions}

In this paper, $\mathrm{Sc}-\mathrm{CO}_{2}$ as a new medium used in the pre-oxidation process of PAN fibers has been studied. FT-IR, DSC, XRD, TG, and density tests were performed to analyze the structural changes of PAN fibers during the cyclization reaction. The results indicated that the degree of cyclization increased with the increase of the cyclization temperature. Comparing the results of PAN fibers treated in two different media, the PAN fibers treated in $\mathrm{Sc}_{-} \mathrm{CO}_{2}$ possessed a higher degree of cyclization than those treated in the air from different test results. The density of the PAN fibers treated in $\mathrm{Sc}-\mathrm{CO}_{2}$ was larger than that in the air.

Author Contributions: M.Q. performed the experiments, analyzed the data and wrote the paper; X.D., Z.H., L.Z. and Y.C. analyzed some of the data; H.K. and M.Y. designed the experiments and revised the paper.

Funding: This work was financially supported by the National Natural Science Foundation of China (Grant No. $51603120,11604204)$, State Key Laboratory for Modification of Chemical Fibers and Polymer Materials, Donghua University (LK1602).

Conflicts of Interest: The authors declare no conflict of interest.

\section{References}

1. Park, S.-J. History and Structure of Carbon Fibers; Springer Nature: Heidelberg, Germany, 2018; pp. 1-30.

2. Park, S.J.; Kim, B.J. Carbon Fibers and Their Composites; Springer Netherlands: Berlin, Germany, 2015; pp. 275-317.

3. Li, H.Y.; Dai, Y.; Lyu, X.F. Application of Carbon Fiber Reinforced Composites on Lightweight Design of Articulated Platform. MSF 2018, 921, 85-90. [CrossRef]

4. Park, S.-J. Novel Carbon Fibers and Their Composites; Springer Nature: Heidelberg, Germany, 2018; Volume 210, pp. 295-342. 
5. Peng, M.; Ma, J. Carbon fiber and its application in light weighting of automobiles. China Synth. Fiber Ind. 2018, 41, 53-57.

6. Wang, Y.Z.; Wang, S.G.; Liu, J.L. Polyacrylonitrile Based Carbon Fibers Obtained from a Melt Spun Route. KEM 2013, 575, 151-155. [CrossRef]

7. Yao, Y.; Chen, J.; Liu, L.; Dong, Y.; Liu, A. Mesophase pitch-based carbon fiber spinning through a filter assembly and the microstructure evolution mechanism. J. Mater. Sci. 2013, 49, 191-198. [CrossRef]

8. Niu, H.; Chang, J.; Liu, W.; Yang, H.; Cao, L.; Zhang, M.; Cao, W.; Wu, D. Structural relationship between random copolyimides and their carbon fibers. J. Mater. Sci. 2016, 52, 1883-1897.

9. Behr, M.J.; Landes, B.G.; Barton, B.E.; Bernius, M.T.; Billovits, G.F.; Hukkanen, E.J.; Patton, J.T.; Wang, W.; Wood, C.; Keane, D.T.; et al. Structure-property model for polyethylene-derived carbon fiber. Carbon 2016, 107, 525-535. [CrossRef]

10. Zhang, X.Y.; Amp, S. Technical Advances and Development Suggestions for Rayon-based Carbon Fiber and Pitch-based Carbon Fiber. Chem. Fert. Des. 2017, 55, 1-3.

11. Frank, E.; Hermanutz, F.; Buchmeiser, M.R. Carbon Fibers: Precursors, Manufacturing, and Properties. Macromol. Mater. Eng. 2012, 297, 493-501. [CrossRef]

12. Aso, H.; Kannabe, T. Polyacrylonitrile-based carbon fiber. TANSO 2007, 2007, 115-121. [CrossRef]

13. Morales, M.S.; Ogale, A.A. Carbon fibers derived from UV-assisted stabilization of wet-spun polyacrylonitrile fibers. J. Appl. Polym. Sci. 2014, 131, 318-323. [CrossRef]

14. Zhao, W.; Lu, Y.; Wang, J.; Chen, Q.; Zhou, L.; Jiang, J.; Chen, L. Improving crosslinking of stabilized polyacrylonitrile fibers and mechanical properties of carbon fibers by irradiating with $\gamma$-ray. Polym. Degrad. Stab. 2016, 133, 16-26. [CrossRef]

15. Zhao, R.-X.; Sun, P.-F.; Liu, R.-J.; Ding, Z.-H.; Li, X.-S.; Liu, X.-Y.; Zhao, X.-D.; Gao, Z.-M. Influence of heating procedures on the surface structure of stabilized polyacrylonitrile fibers. Appl. Surf. Sci. 2018, 433, 321-328. [CrossRef]

16. Liu, J.; Xiao, S.; Shen, Z.; Xu, L.; Zhang, L.; Peng, J. Study on the oxidative stabilization of polyacrylonitrile fibers by microwave heating. Polym. Degrad. Stab. 2018, 150, 86-91. [CrossRef]

17. Fitzer, E.; Frohs, W.; Heine, M. Optimization of stabilization and carbonization treatment of PAN fibres and structural characterization of the resulting carbon fibres. Carbon 1986, 24, 387-395. [CrossRef]

18. Banerjee, T.; Lipscomb, G.G. Direct measurement of the carbon dioxide-induced glass transition depression in a family of substituted polycarbonates. J. Appl. Polym. Sci. 1998, 68, 1441-1449. [CrossRef]

19. Elmaaty, T.A.; El-Taweel, F.; Okubayashi, S.; Ma, J.; El-Aziz, E.A. Facile Bifunctional Dyeing of Polyester under Supercritical Carbon Dioxide Medium with New Antibacterial Hydrazono Propanenitrile Dyes. Ind. Eng. Chem. Res. 2014, 53, 15566-15570. [CrossRef]

20. Zheng, H.; Zhang, J.; Yan, J.; Zheng, L. An industrial scale multiple supercritical carbon dioxide apparatus and its eco-friendly dyeing production. J. CO2 Util. 2016, 16, 272-281. [CrossRef]

21. Zheng, H.-D.; Zhang, J.; Yan, J.; Zheng, L.-J. Investigations on the effect of carriers on meta-aramid fabric dyeing properties in supercritical carbon dioxide. RSC Adv. 2017, 7, 3470-3479. [CrossRef]

22. Friedrich, J.P.; Pryde, E.H. Supercritical CO2 extraction of lipid-bearing materials and characterization of the products. J. Am. Oil Chem. Soc. 1984, 61, 223-228. [CrossRef]

23. Benito-Román, Ó.; Rodríguez-Perrino, M.; Sanz, M.T.; Melgosa, R.; Beltran, S. Supercritical carbon dioxide extraction of quinoa oil: Study of the influence of process parameters on the extraction yield and oil quality. J. Supercrit. Fluids 2018, 139, 62-71.

24. Bonthuys, G.J.K.; Schwarz, C.E.; Burger, A.J.; Knoetze, J.H. Separation of alkanes and alcohols with supercritical fluids. Part I: Phase equilibria and viability study. J. Supercrit. Fluids 2011, 57, 101-111. [CrossRef]

25. Clifford, A.A. Reactions in Supercritical Fluids; Springer Netherlands: Berlin, Germany, 1994; pp. 449-479.

26. Dudnik, A.O.; Trofimchuk, E.S.; Efimov, A.V.; Nikonorova, N.I.; Rukhlya, E.G.; Nikitin, L.N.; Yaminsky, I.V.; Volynskii, A.L. Evolution of the Nanoporous Structure of High-Density Polyethylene during Drawing in Supercritical Carbon Dioxide. Macromolecules 2018, 51, 1129-1140. [CrossRef]

27. Rebocho, S.; Cordas, C.M.; Viveiros, R.; Casimiro, T. Development of a ferrocenyl-based MIP in supercritical carbon dioxide: Towards an electrochemical sensor for bisphenol A. J. Supercrit. Fluids 2018, 135, 98-104. [CrossRef] 
28. Johns, A.I.; Rashid, S.; Watson, J.T.R.; Clifford, A.A. Thermal conductivity of argon, nitrogen and carbon dioxide at elevated temperatures and pressures. J. Chem. Soc. Faraday Trans. 1 1986, 82, 2235. [CrossRef]

29. Kouta, A.; Al-Sulaiman, F.; Atif, M.; Bin Marshad, S. Entropy, exergy, and cost analyses of solar driven cogeneration systems using supercritical CO2 Brayton cycles and MEE-TVC desalination system. Energy Convers. Manag. 2016, 115, 253-264. [CrossRef]

30. Sengers, J.; Michels, A.; Van Der Gulik, P. The thermal conductivity of carbon dioxide in the critical region: I. The thermal conductivity apparatus. Physica 1962, 28, 1201-1215.

31. Le Neindre, B. Contribution a l'etude experimentale de la conductivite thermique de quelques fluides a haute temperature et a haute pression. Int. J. Heat Mass Transf. 1972, 15, 1-24. [CrossRef]

32. Stephan, K.; Laesecke, A. The Thermal Conductivity of Fluid Air. J. Phys. Chem. Ref. Data 1985, 14, 227. [CrossRef]

33. Lin, X.; Wang, C.G.; Yu, M.J.; Lin, Z.T.; Zhang, S. Study on the Local Structure of PAN-Based Carbon Fiber Using Radial Distribution Function Based on XRD. AMR 2013, 664, 614-619. [CrossRef]

(C) 2019 by the authors. Licensee MDPI, Basel, Switzerland. This article is an open access article distributed under the terms and conditions of the Creative Commons Attribution (CC BY) license (http:// creativecommons.org/licenses/by/4.0/). 